Projets

de paysage

\section{Projets de paysage}

Revue scientifique sur la conception et l'aménagement de l'espace

19 | 2018

L'imagerie du paysage

\title{
Regarder, représenter, comprendre
}

Regarder les paysages, représenter la diversité, faire comprendre et partager le projet de paysage

Looking, Representing, Understanding: Looking at Landscapes, Representing

Diversity, Understanding and Sharing the Landscape Project

\section{Florence Robert et Frédéric Bœuf}

\section{OpenEdition}

\section{Journals}

Édition électronique

URL : http://journals.openedition.org/paysage/315

DOI : 10.4000 /paysage.315

ISSN : 1969-6124

\section{Éditeur :}

École nationale supérieure du paysage de Versailles-Marseille, Institut national des sciences appliquées Centre Val de Loire - École de la nature et du paysage, École nationale supérieure d'architecture et de paysage de Bordeaux, École nationale supérieure d'architecture et de paysage de Lille, Agrocampus Angers

\section{Référence électronique}

Florence Robert et Frédéric Bœuf, «Regarder, représenter, comprendre », Projets de paysage [En ligne] 19 | 2018, mis en ligne le 01 décembre 2018, consulté le 28 novembre 2019. URL : http:// journals.openedition.org/paysage/315 ; DOI : 10.4000/paysage.315

Ce document a été généré automatiquement le 28 novembre 2019.

Projets de paysage 


\section{Regarder, représenter, comprendre}

Regarder les paysages, représenter la diversité, faire comprendre et partager le projet de paysage

Looking, Representing, Understanding: Looking at Landscapes, Representing

Diversity, Understanding and Sharing the Landscape Project

Florence Robert et Frédéric Bœuf

1 Paysagistes depuis plus de 20 ans nous travaillons sur divers projets avec différents partenaires, en équipes restreintes ou plus étoffées, sur des sites très urbains ou plus ruraux, dans différentes villes, différentes régions. Nous parcourons la France au hasard des commandes et des engagements pour réaliser jardins, aménagements, analyse de territoire, mais toujours autour de l'espace public ou partagé. Notre sensibilité nous amène à reconnaître les paysages et leurs diversités, à nous en inspirer aussi. Notre fascination pour le monde du végétal pousse notre curiosité dans une recherche de solutions esthétiques, économiques et pérennes. Notre travail se double d'une recherche sur la représentation d'une flore plus indépendante des jardiniers, moins horticole, peut-être moins appréciée, et pourtant expressive.

Faire avec la nature, plutôt que contre, nous oblige à comprendre les dynamiques des écosystèmes, à changer notre regard pour apprécier une végétation plus autonome, plus « sauvage».

contact[at]rb-cie[dot]net, www.rb-cie.net

Paysagistes concepteurs, notre travail est largement illustré de documents graphiques et techniques permettant la compréhension du projet (aménagement de places, de jardins ou d'espaces publics) et la visualisation des attendus paysagers.

Dans ce cadre, nous sommes amenés à produire des images. Pour cela, nous cherchons l'inspiration en amont des projets. Comme le botaniste collectionne les herbes et les plantes qu'il trouve, nous collectionnons les paysages parcourus, traversés ou analysés. Cette collecte donne lieu a des « herbiers de paysage » mêlant données prises sur le vif (photos, croquis, feuilles) et images recomposées. C'est ce travail sur les données brutes et données transformées qui structure notre part d'invention dans le projet. 


\section{La prolifération des images}

4 La production d'images est un incontournable. Pour expliquer, décrire, séduire il faut présenter des images. La concurrence aidant, la surenchère est là. Dans la grande fanfare des couleurs et des matières, comment faire entendre son petit air de flûte ? Les visuels saturent les réflexions, emportent toutes les préoccupations. On en oublierait qu'un espace c'est aussi une pratique du lieu.

\section{Le formatage de l'outil informatique}

5 Pour répondre à cette exigence, nous avons aujourd'hui de nombreux outils : logiciels de dessin, appareils photo, banque d'images. Mais hélas tout le monde a les mêmes. Les outils ne permettent plus de faire la différence. Cette mise à disposition de moyens très accessibles crée un consensus, une mode. Toutes les images se ressemblent: cristallines, couleurs saturées, beaucoup de vert. Dans le brouillard de la technique de la représentation, il devient difficile de percevoir la pertinence du projet.

\section{La facilité du copier/coller}

Les images circulent, on se les échange, on se les «bidouille», on se les «vole». La reproduction et la compilation sont devenues tellement aisées. Nous ne savons plus vraiment qui a fait quoi. Les plans, les coupes, les perspectives et les images de référence sont devenus de véritables patchworks retravaillés, recopiés d'un projet sur l'autre.

\section{Comment garder la maîtrise?}

7 Il nous faut prendre un peu de hauteur, retrouver une sérénité. Il nous est nécessaire de nous dégager de cette obsession, dégager nos yeux de la captation des écrans et retrouver la réalité du lieu.

\section{Représenter et comprendre}

8 Photographier un lieu, redessiner le plan d'un espace, croquer un paysage, c'est apprendre comment il est composé et en même temps garder une mémoire reproductible du lieu.

9 Au hasard de nos contrats de maîtrise d'œuvre, nous explorons les régions, leurs paysages, leurs écosystèmes. C'est l'occasion pour nous d'arpenter, d'observer ces marais, ces forêts, ces prairies sèches, ces estuaires, ces friches urbaines... Nous photographions, dessinons, collectons substrat et végétaux. De retour à l'atelier, nous classons, archivons, mais également retravaillons nos images et dessins, nous scannons nos collectes. Pour garder trace de cette promenade, nous fabriquons, sous forme de compilations/sélections des petits cahiers $20 \times 20 \mathrm{~cm}$. À l'occasion de nouveaux projets, cette base de données est consultée pour nous inspirer de nouvelles idées. Ces notes et visuels nous permettent également d'illustrer nos présentations, que ce soit au sien de 
l'agence ou avec la maîtrise d'ouvrage lors des réunions de travail et/ou de concertation. Ces petits cahiers $20 \times 20$ sont présentés sur la table de travail et permettent à nos partenaires de mieux saisir nos intuitions. Ils apportent une note sensible à notre savoir-faire autour de l'élaboration de projet.

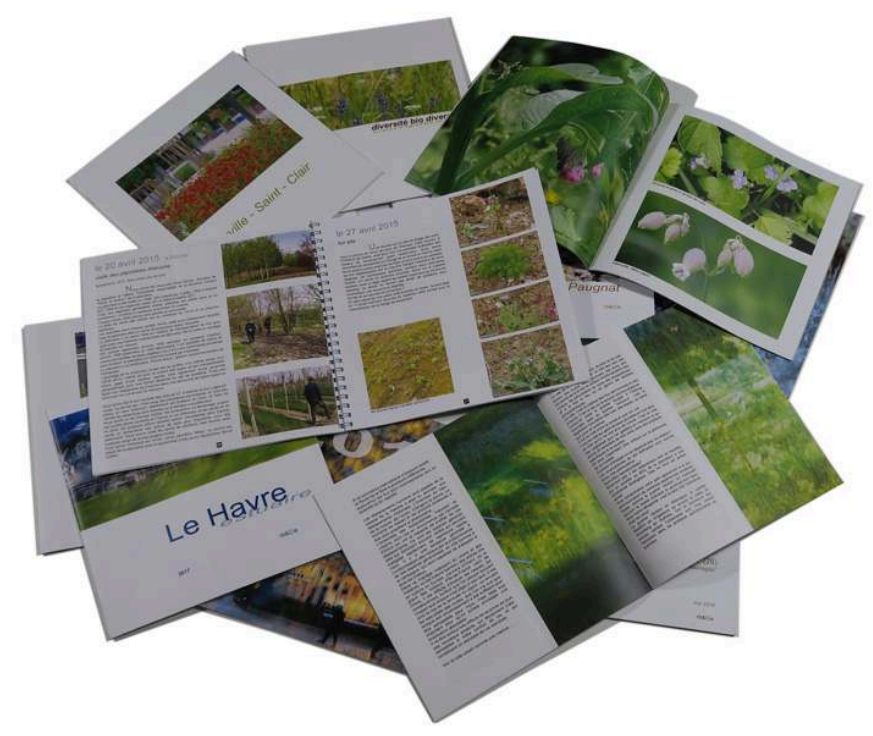

Cette démarche d'abord intuitive s'est peu à peu fixée comme un véritable travail à part entière, en parallèle de notre travail de concepteur. Comme un pianiste fait ses gammes, nous arpentons des lieux de nature, pour archiver de la donnée, apprendre à comprendre comment ces sites s'organisent, apprendre à regarder, à reconnaître. L'appareil photo et le carnet sont nos outils pédagogiques.

11 Depuis peu, dans le cadre de nos projets, nous invitons nos partenaires, donneurs d'ordre, gestionnaires, habitants à ces promenades découvertes. Nous les déclinons également sous la forme de cahiers spécifiques ou d'expositions photos, pour partager cet apprentissage du regard et de l'observation.

\section{La préparation en amont des projets}

12 L'intendance des projets de commande est toujours contrainte par un calendrier serré. Il faut être capable de produire dans des délais courts. La tentation est grande de reprendre concepts et présentations des projets précédents pour satisfaire cette exigence, surtout si ces projets sont réussis. La visite régulière de sites naturels renouvelle les idées et les inspirations. Examiner de nouveaux assemblages, de nouvelles structures en amont de la pression des projets est un temps pour la réflexion, l'observation. C'est un temps également pour progresser dans la prise de note, la prise de vue. De retour à l'atelier, archiver, classer les photos, déclenche un renouvellement de notre imaginaire qui sera prêt pour un nouveau déploiement lors du prochain projet de commande. 

labyrinthes, dont nous ne maîtrisons généralement qu'une infime partie. Devant la complexité de ces outils, on se limite à ce que l'on sait déjà faire. Pour éviter la perte de temps, on reprend ce que l'on a l'habitude de produire. Avec ce nouveau stock d'images récoltées lors de ces visites, la sérénité est suffisante pour expérimenter des techniques originales de détourage, de déformation, d'assemblage, pour utiliser de nouvelles palettes d'outil, de couleurs, de matières. C'est le moment calme pour préparer nos fonds, nos aplats, nos textures qu'on utilisera dans le prochain projet.

Plus qu'un véritable travail d'archivage et de classement qui se fait de toute façon, c'est surtout un exercice indispensable pour développer nos envies, nos aspirations qui nous pousseront à faire autrement. Dans l'urgence de la commande de projet, on n'a pas le temps de se demander pourquoi on élabore une image. Le programme impose un plan : on trace un plan, le contrat exige deux coupes et une perspective: on dessine deux coupes et une perspective. Dans le monde du bâtiment et travaux publics (BTP) la description de projet se définit dans la fabrique de dossier très normé et inspiré par la matière inerte : le béton, l'acier, les murs, le sol... Il nous est difficile dans ce contexte de décrire sans déroger à la règle le dynamisme des structures végétales et leurs évolutions saisonnières... Un arbre c'est, au début de sa végétation, une graine, puis un baliveau de deux mètres de haut et plus tard encore un sujet avec un houppier de dix mètres de diamètre. Comment traduire cette diversité de forme, cette évolution en taille et volume en un seul plan? Un bosquet c'est un parterre de plants forestiers à la plantation puis une forêt à maturité : comment en dessiner l'élévation? Toutes ces questions ne peuvent être abordées dans le cadre du projet. Alors pour rester dans le planning, on utilise le cadre proposé en oubliant la spécificité du vivant. Le vivant ça grandit, ça évolue, ça n'entre pas forcément dans les cases. Ce travail de visite, de collecte de données, en amont des projets, nous permet de mieux appréhender ce hors cadre BTP qui nous permettra de mieux faire passer nos idées. C'est là le paradoxe : nos partenaires attendent de nous que nous intervenions sur cette notion de nature, du vivant, mais trop souvent l'espace de travail qui nous est accordé ne le permet pas !

Ce paradoxe s'exprime de façon caricaturale avec les types de logiciel adoptés. Les acteurs orthodoxes du BTP utilisent des logiciels qui dessinent au trait. Ces traits sont générés par le calcul, ce qui supprime toute trace de l'opérateur et donne l'apparence d'un point de vue neutre. Ces outils vectoriels travaillent sur la définition des limites. Nos réflexions nous ont conduits à préférer les logiciels qui expriment le pixel, qui eux gardent trace de l'opérateur. Comme l'appareil photo permet à l'utilisateur de faire transparaître son style, son point de vue, ces logiciels du pixel transcrivent de façon sensible matière et couleur, valeurs incontournables du végétal. L'expression des limites en matière de massifs de plantes ou de paysage n'est pas, de notre point de vue, pertinente.

16 Visiter les paysages et appréhender leurs écosystèmes, les photographier, est un moment de recul par rapport à la pression de la fabrique des images à l'intérieur du projet. Ce recul nous permet de mieux nous positionner face à l'acte de création des mises en espace de ce projet. 


\section{L'image comme une incitation à arpenter le site}

17 Prendre une photographie oblige à aller sur place. Faire une photo impose de choisir un point de vue, un cadrage. Tout notre travail sur la représentation tourne autour de ces allers-retours sur les lieux. Saisir du regard l'existant sur lequel nous allons inventer, explorer les sites voisins, observer les sites similaires, parcourir les espaces de nature et en ramener des visuels, des traces que nous compilons, assemblons, découpons, pour projeter nos rêves de transformation. Nous ne partons pas de la feuille blanche, nous sommes dans la perception d'une réalité en évolution. Même nos dessins techniques, les plans comme les coupes, sont des "photos » du site, des sites, retravaillées certes, même souvent très manipulées, mais il y a toujours en toile de fond cette trace d'un existant.

En fait notre véritable plaisir est dans le déplacement : voir des lieux, en ramener des images pour mieux montrer ce que l'on a vu et inciter nos partenaires à se rendre sur place à leur tour. La nature accomplit très bien toute seule les transformations et, bien souvent, nous ne sommes là que pour stimuler, contempler et faire contempler.

\section{Un point de vue pour révéler les invisibles}

19 Faire une image, choisir un point de vue, retenir des outils pour concevoir cette image, c'est créer des biais qui contraignent la perception.

Dessiner en noir et blanc n'est pas pareil que dessiner en couleurs, tracer au trait n'est pas pareil que peindre en aplat, ébaucher une perspective n'est pas pareil que lever un géométral... représenter avec une règle c'est fatalement ne percevoir que des solutions sur la ligne droite... Le choix des outils de représentation doit se faire en conscience.

21 Notre point de vue, notre «biais ", c'est de faire surgir les deuxièmes plans, ce qui est derrière, dessous, ce qui n'apparaît pas au premier regard. Le végétal est un levier. Prendre des photos au macro permet de montrer que la ronce fait aussi une fleur délicate, qu'entre les pétales des araignées tissent leur toile. Le vivant est multiple, complexe et il génère de formidables capacités d'émerveillement.

Lorsque l'on nous demande d'intervenir sur un site, c'est qu'il y a un désordre, une «mauvaise " perception. Il paraît alors facile de tout transformer et de tout replanter. Mais dans une démarche économe nous préférons changer notre regard, percevoir ce qui se cache sous cette surface soi-disant détériorée, révéler la beauté de la nature qui se tisse sous ces apparents dysfonctionnements : au cœur du triste sous-bois il y a le fleurissement des perce-neige. Voilà ce que nous cherchons à montrer.

\section{Des images autonomes}

Dans la fabrique des projets de commande, la production d'images, plans, détails, illustrations de palettes végétales... est dédiée à la description d'un lieu et aux propositions d'aménagement. Ce sont des images servantes. Elles montrent ce qui est, ce qui sera.

Notre recherche patiente en amont des projets nous a permis de développer d'autres stratégies, d'autres ambitions. Dégagées de tout contexte programmatique, les images 
ainsi produites atteignent une certaine autonomie. Elles peuvent être contemplées pour la seule émotion qu'elles suscitent, sans renvoyer à une nécessité de production : une transformation à opérer, un projet de paysage à développer.

Il y a le plaisir de faire des images, de tripatouiller les pixels ou le pigment, le plaisir du geste qui trace sur le papier, d'entrer dans l'arborescence du logiciel, de faire chanter la couleur ou la graphie, d'extraire la matière image. C'est le plaisir que nous découvrons dans le secret de notre atelier.

Mais il y a également le plaisir d'offrir aux regards des autres notre production, de voir leurs réactions, d'observer les débats suscités. Des images telle une "mise en œil ", comme on dit « une mise en bouche », pour préparer nos partenaires de projet au plat principal : l'acte de conception que nous allons devoir faire ensemble.

Au-delà de la description, l'image peut émouvoir. Lorsque nous arrivons à provoquer chez nos partenaires (bureau d'études, architectes, maîtres d'ouvrages, associations, habitants) cet état émotionnel, sensible, ils se trouvent plus enclins à observer la complexité. Ils en viennent à accepter l'incontrôlable, la diversité du monde végétal, à en être moins inquiétés aussi. Une écoute plus attentive aux questions environnementales s'installe.

Le projet gagne là sa principale ambition: attirer l'attention sur les questions environnementales, et non sur la forme du jardin.

\section{Le flou}

Nous utilisons des images lors de l'élaboration de projets d'espaces publics pour échanger avec les futurs gestionnaires, les jardiniers, les habitants et les autres partenaires.Les illustrations sont une invitation au débat avec nos interlocuteurs, pour tester nos idées, enrichir notre invention.

Mais lorsque l'on présente une photo, d'un arbre par exemple, on donne tout de suite à voir quel type d'arbre c'est (sa taille, son houppier, sa situation dans un espace précis). On peut lire tout de suite s'il est grand et trop grand, trop haut ou pas assez. Il y a là une difficulté à ne pas pouvoir avancer pas à pas avec des images car elles donnent souvent tout de suite une définition trop précise des choses montrées. C'est pour cette raison que même la photo la plus simple est toujours retravaillée, recadrée, pour éliminer les idées parasites ou trop élaborées. C'est pour cela que le dessin est souvent plus performant que la photo : le tri de ce que l'on montre se fait instinctivement.

Nous utilisons le flou car c'est un moyen simple pour gommer sans tout enlever. C'est dans l'espace du flou que nos interlocuteurs peuvent avancer leurs propres idées. $\mathrm{Ne}$ pas finir complètement la description tout en laissant quelques traces/guides permet de continuer le dialogue, d'envisager des modifications possibles, des évolutions. Ce flou permet également que le projet nous échappe à un certain moment, qu'il soit rendu aux usagers. Comme une déviance qui permet aux plantations d'être autonomes, que la vie prenne le dessus. Le flou est cet espace de libération. Le projet de jardin, comme celui de l'espace public, est toujours inachevé, toujours en train de se faire, suivant les usages, les saisons... toujours un peu flou... comme si la prise de vue photographique, avec une vitesse trop lente, n'avait pas permis de saisir le sujet mobile et avait imprimé, sur la plaque sensible, un filé, un flou. 


\section{Quelques exemples}

La prairie aux Totems, aménagement paysager pour un collectif d'habitation à Colombelles (14)
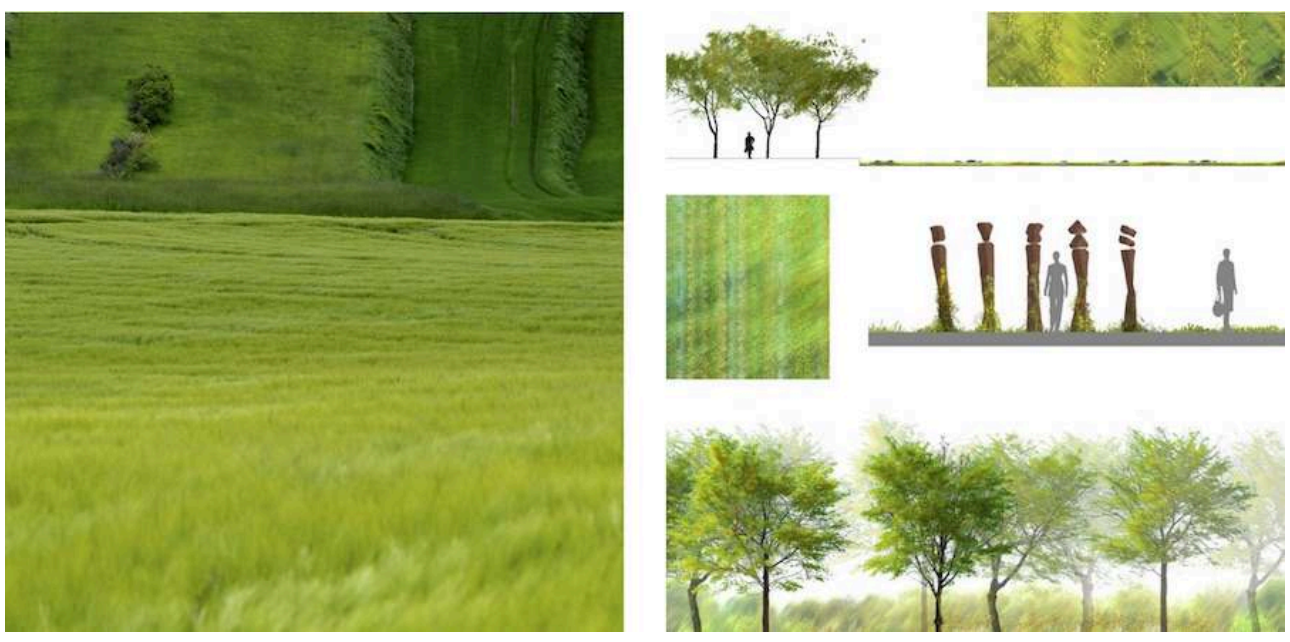

À gauche, image de référence, champ agricole ; à droite, visuel du projet, esquisse automne 2010.

La photo du champ, prise lors d'une de nos déambulations dans la campagne de Seineet-Marne, remaniée à la manière d'une vue perpective/plan et les effets du moutonnement vert clair des blés nous inspirent pour tenter de reproduire cette fraîcheur du champ en ville. Pour le projet de Colombelles, et dans le cadre du travail de résidentialisation, nous avons opté pour une prairie haute à la place des gazons nus. Les dessins du projet s'inspirent naturellement de l'effet tapis des blés.

Square du Château du Loir à Courbevoie (92)
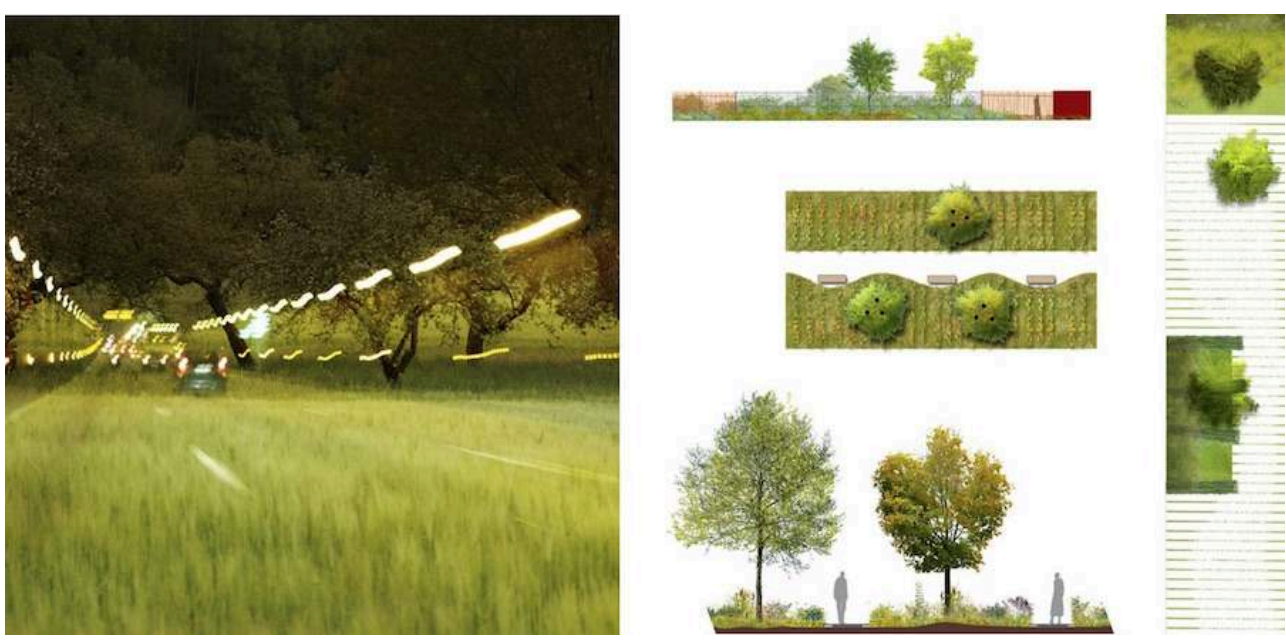

À gauche, photomontage, image de référence ; à droite, visuel du projet, détail, plan et coupes, 2011.

À gauche un travail spécifique sur la lumière la nuit en ville et des éclairages : photo revue et "renaturée » à l'extrême (la route disparait sous les prés). C'est un peu une manière d'imaginer comment se déplacer dans une nature présente dans la ville. Pour le projet de Courbevoie, les cheminements piétons en sol imperméable sont limités. Les 
arbres ponctuent l'aménagement de façon aléatoire. Pas d'alignement, ce qui répond mieux aux fonctions des espaces ouverts et accessibles.

Concours pour l'écoquartier de la Marine à Colombe (92)
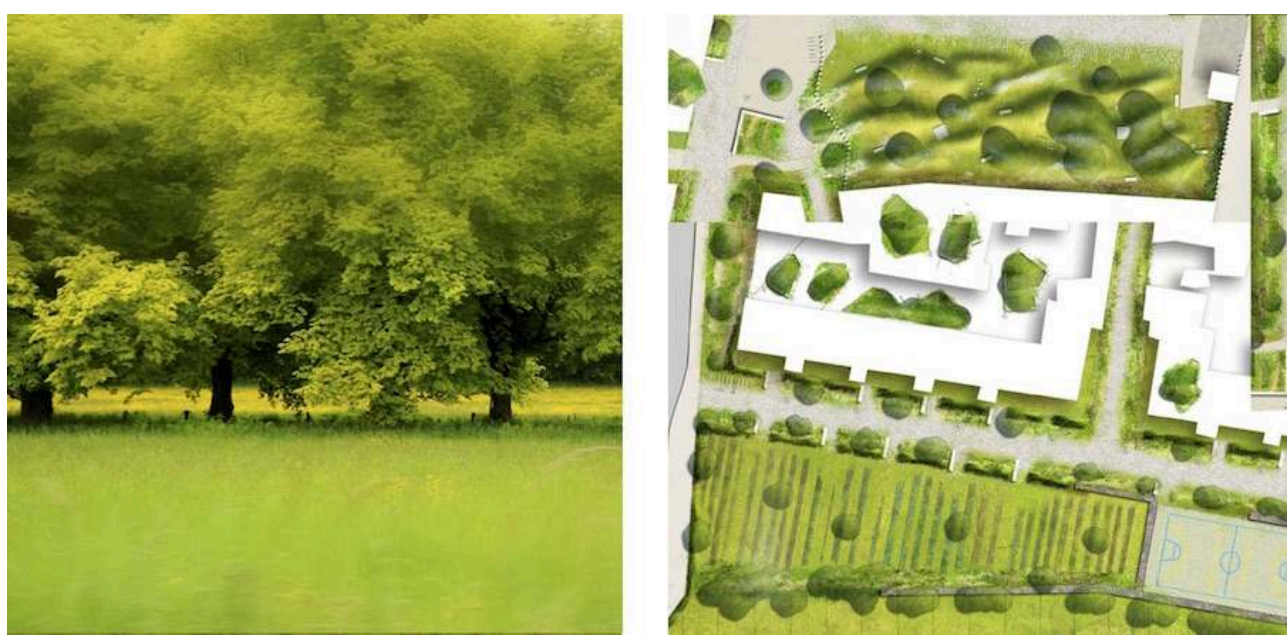

À gauche, photomontage, image de référence ; à droite, plan paysage des aménagements extérieurs 2011.

Notre travail de concepteur tend à imaginer les espaces publics avec le plus de sols perméables et végétalisés possibles. Les associations végétales observées lors de nos voyages de recherche nous invitent à déceler des organisations intéressantes. Ici, cette photo de champs et d'arbres retravaillée afin d'exacerber le contraste lumineux entre champs clairs et houppiers sombres comme des nuages flottant au-dessus du sol. Des arbres formant lisière et masque, îlot vertical dans un océan de planéité. Pour le projet d'écoquartier de Colombes, nous avons organisé le jardin central en dunes et bosquets d'arbres, plissant le sol mais gardant l'idée de contraste fort entre les structures verticales et souples des arbres et basses du tapis végétal ras. La dominante colorée du vert jaune de la photo retravaillée nous sert de modèle et d'étalonnage pour finaliser la palette colorée de notre travail.

Aménagement des espaces extérieurs, place Braque à La Courneuve (93)
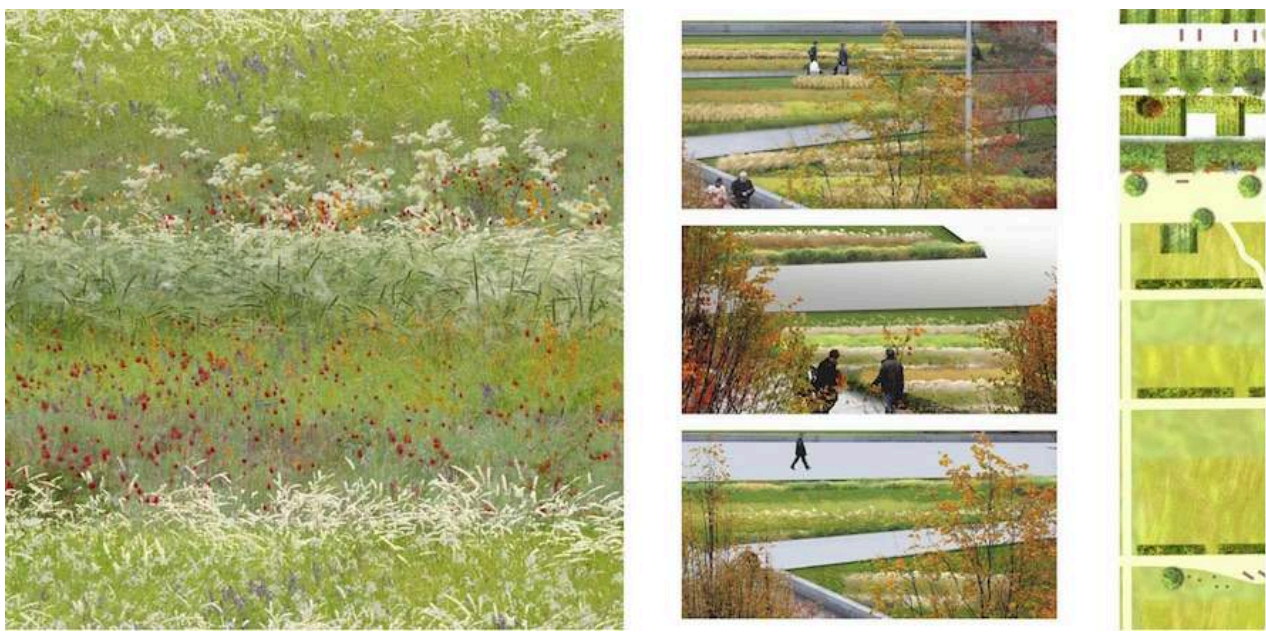

À gauche, recherche de matières ; à droite, visuel du projet, perspective et plan 2011.

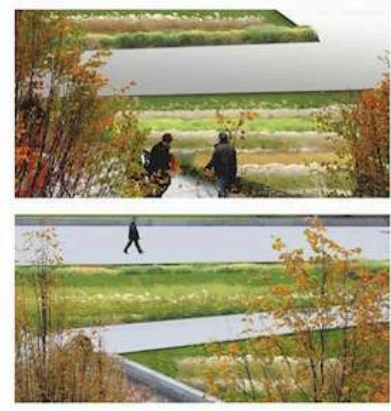


À partir des photos réalisées au cours des nombreuses ballades, nous avons réuni une iconographie importante de matières végétales: champs cultivés, prairies sauvages, prairies sèches ou humides, structures basses ou hautes. À partir de ces données brutes, un travail de superposition et d'association est mené pour nous donner des outils graphiques propres à interpréter nos projets de végétalisation des sols en ville. De même que la nature sauvage nous inspire par sa richesse d'associations et surtout de mélange des formes et des structures végétales, nous réfléchissons à des associations végétales qui par leur structure graphique peuvent apporter une originalité et une richesse esthétiques aux plantations urbaines. De cette recherche esthétique, nous utilisons les images comme fond dans le cadre du dessin du projet de paysage. Ici des croquis et/ou des perspectives permettent de montrer dans une phase avant-projet les ambiances qui pourraient être déclinées.

Recherche sur la perméabilité des sols, 2012
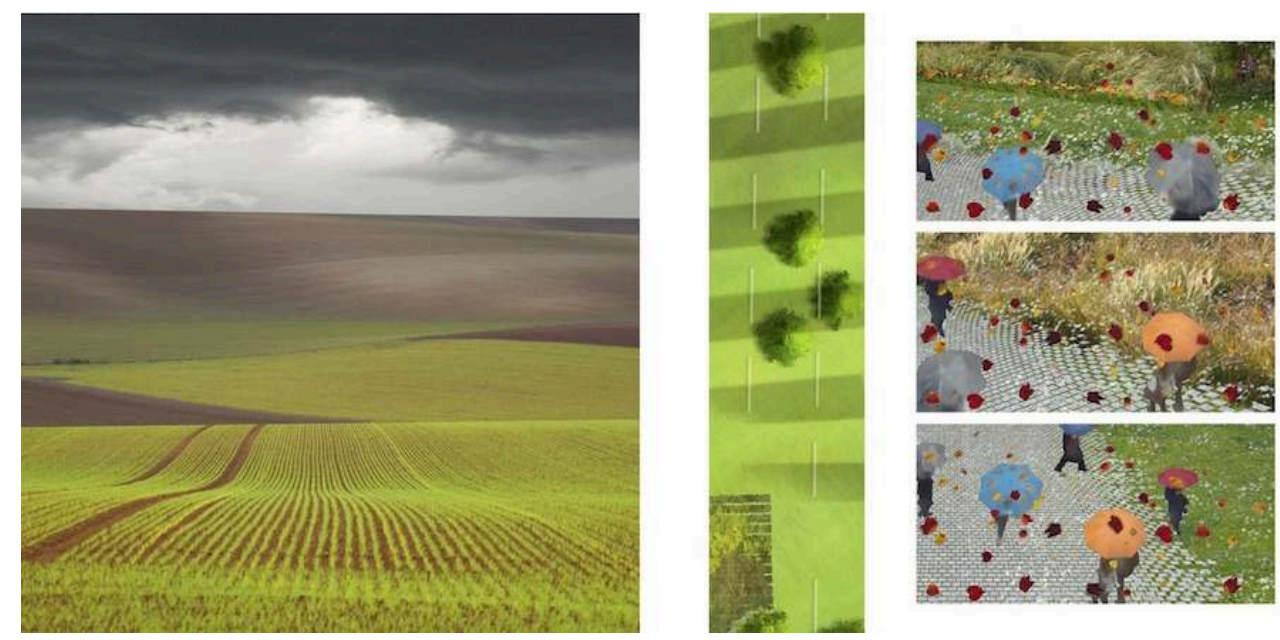

À gauche, photomontage, structure de sol agricole ; à droite, visuels de sols urbains.

Représenter graphiquement le sol et sa perméabilité n'est pas toujours très facile. À partir des photos diverses de champs, nous tirons de façon quasi instinctive des impressions, des illusions optiques (ici les rayons des semis qui font miroiter le sol) que nous réinterprétons dans les dessins du projet (dans les plans, mais aussi les perspectives).

Cette vue retravaillée de champ agricole apporte également une proposition de composition d'image : la partie champ au $2 / 3$ et le ciel sur $1 / 3$. Cette mise en image est souvent source d'idées pour l'organisation et le montage du rendu de nos images «projet». Mettre trois croquis perspectifs sur une page est plus dynamique qu'en mettre un seul. Il y a une idée de mouvement qui s'installe dans le document statique du support papier. 

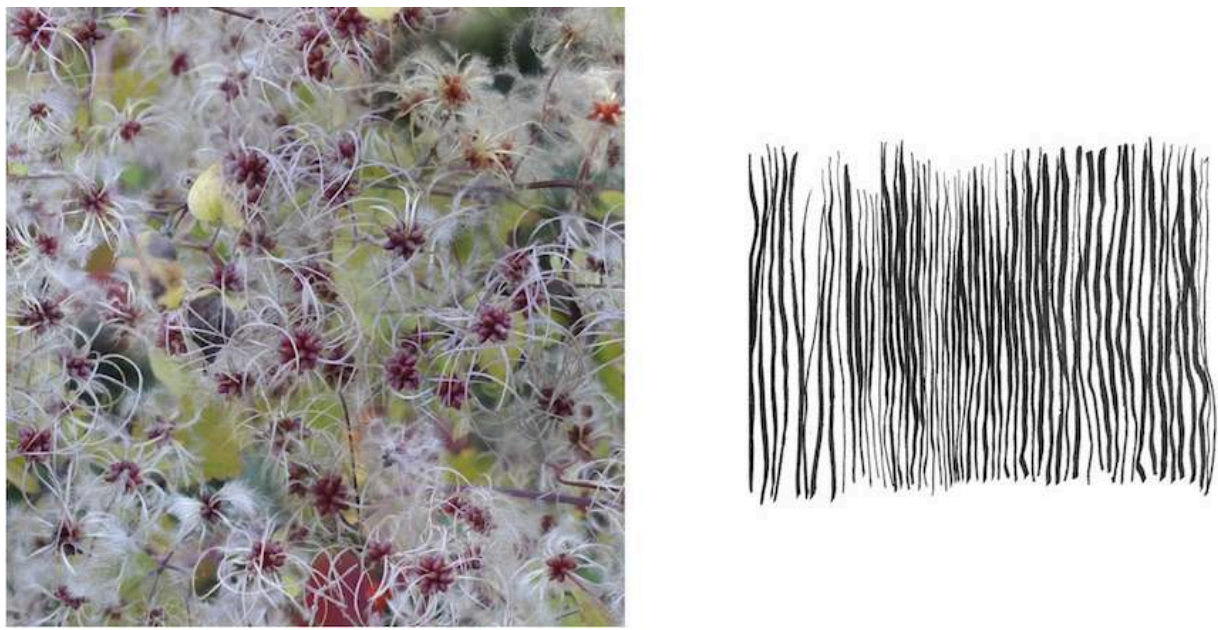

À gauche, clématite en graine - photomontage ; à droite, rythme au pinceau japonais.

La recherche patiente est un moyen ludique de découvrir aussi bien la beauté sobre des plantes que les effets graphiques du pinceau japonais. C'est un travail qui combine la réflexion sur les rythmes, la densité du trait, l'aplat du montage. Il nous permet de conserver une certaine dextérité et une agilité à traduire des moments, des idées, des intentions.
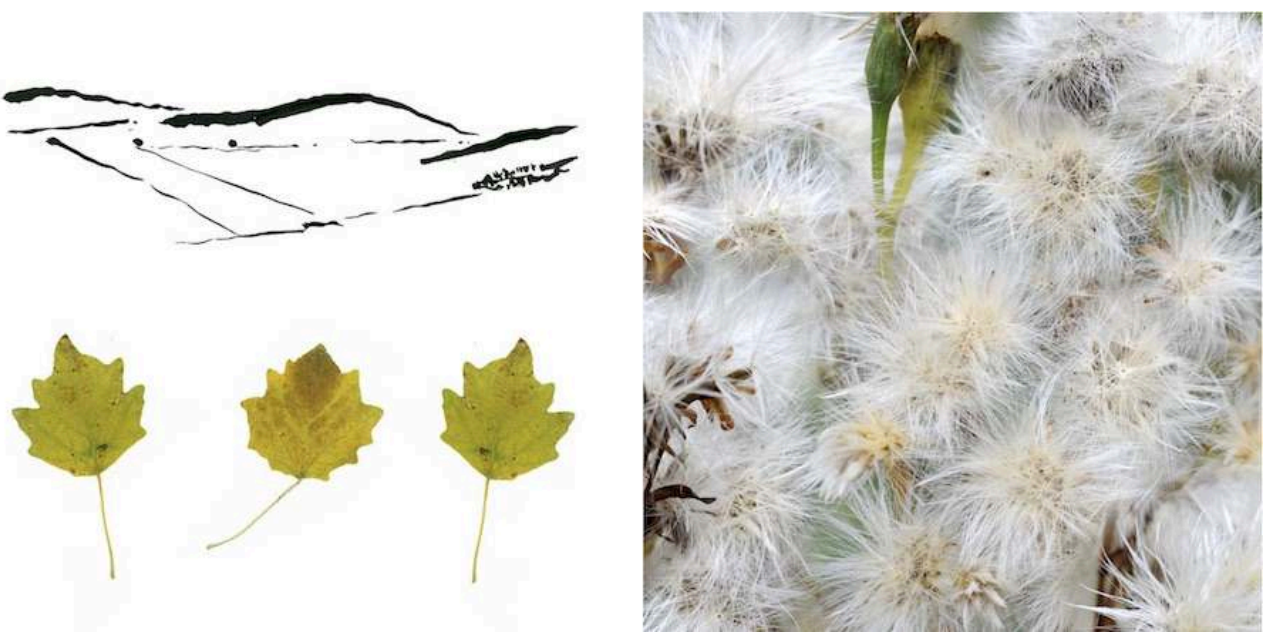

À gauche, paysage de dune et feuilles de peuplier ; à droite, aster maritime fané - photomontage.
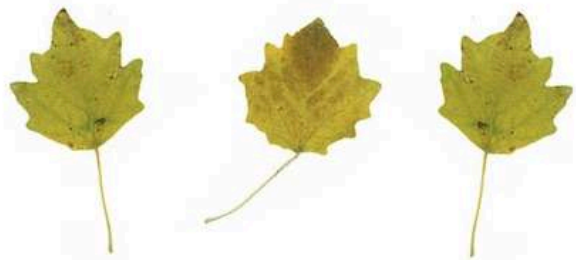

Croquer sur le vif le mouvement du relief, savourer le délié d'une vallée, tracer les lignes de fuite pour appréhender l'organisation du paysage, prélever des feuilles tombées au sol et comprendre la grande diversité de formes des feuilles d'un même arbre...

Parallèlement au travail graphique manuel, l'œil photographique capte des formes infiniment fines que le travail à l'atelier accentue et met en scène pour valoriser et donner à voir les petites choses du bord du chemin. 

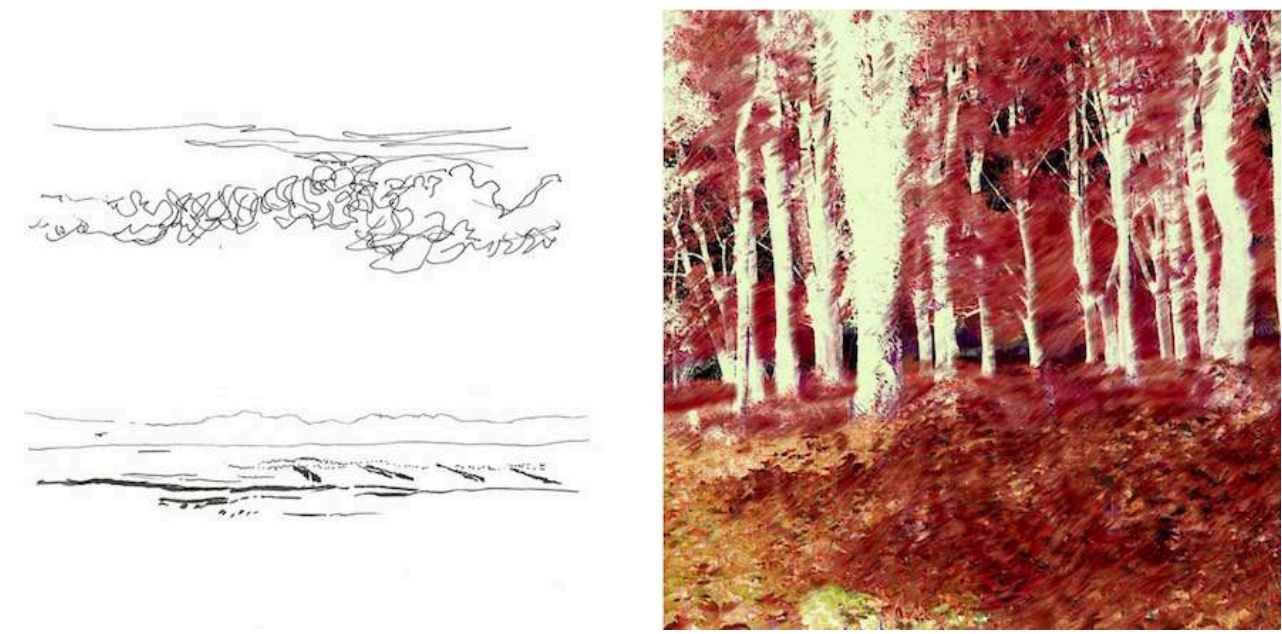

À gauche, paysage sous les nuages : lignes du ciel et lignes des eaux, Berck Plage ; à droite, abandon du vert, marais de la Brenne (36).

Chaque site et chaque voyage apportent son lot de traces que nous compilons inlassablement dans nos carnets. Rendus à l'atelier, nous produisons des images comme sublimées du voyage, pour faire rêver... Elles servent de support esthétique à notre vision poétique de la réalité. Pour faire un pas de côté et voir l'insaisissable... des troncs blancs dans une forêt rouge.

\section{Recherche patiente, 2016}
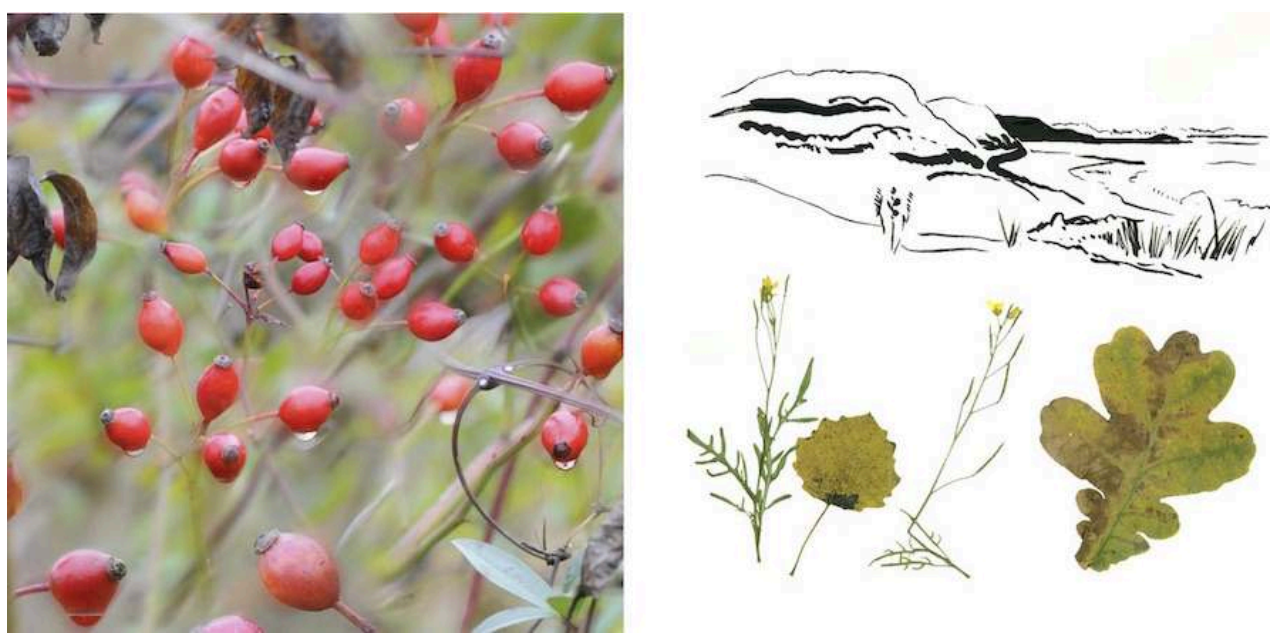

À gauche, cynorhodons après la pluie - photomontage ; à droite, paysage de la baie d'Authie (62) et collecte de feuilles et d'herbes...

Repérer les couleurs vives de la nature, en faire collection... Dessiner les structures géologiques du paysage et faire herbier de tout; des arbres du site et des herbes sauvages qui poussent en travers des chemins. Cette collecte est comme un apprentissage cent fois répété de ce que la nature offre gracieusement : herbes, feuilles, arbres, humus, petits fruits pour les oiseaux, feuilles et litières pour les petits animaux... 
Recherche patiente, 2016
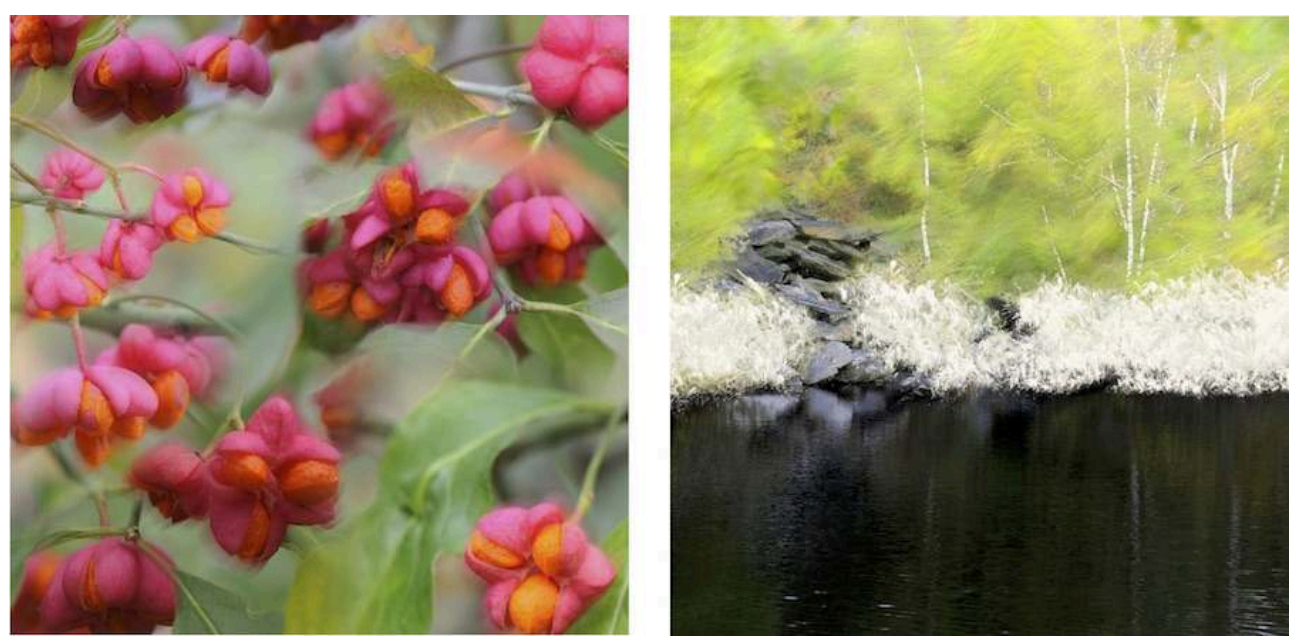

À gauche, graines du fusain d'Europe Euonymus europaeus - photomontage ; à droite, terres noires, ancienne ardoisière de Trélazé (49)

Dans le lointain, des couleurs vives scintillent: ce sont les baies du fusain d'Europe, arbuste indigène en France.

À l'opposé, les terres noires de Trélazé et ses ardoisières créent un sol lunaire spécifique : drainant, et très pauvre... seules les plantes particulièrement pionnières peuvent s'y installer... Et en automne, le feuillage doré des bouleaux s'accorde à merveille avec les lacs noirs des anciennes fosses d'extraction. Quand la nature nous donne des leçons de colorimétrie... et démontre son pouvoir de régénération.

Recherche patiente, 2017
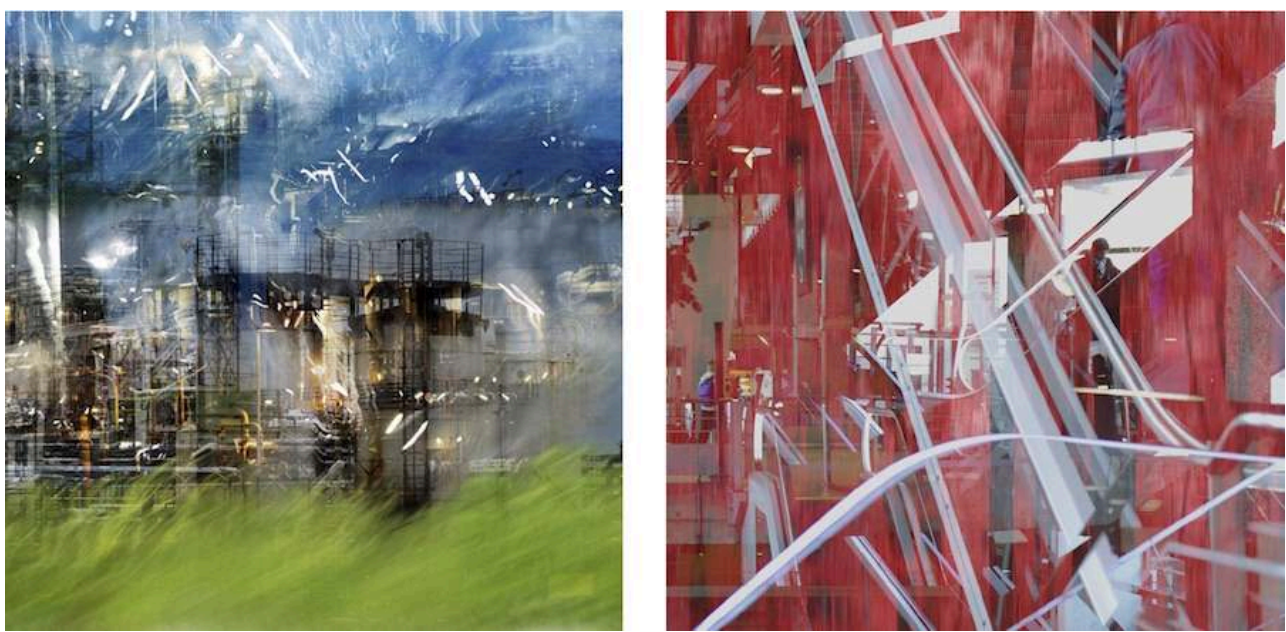

À gauche, paysage industriel vs zone naturelle, Le Havre (76) ; à droite, infrarouge. 

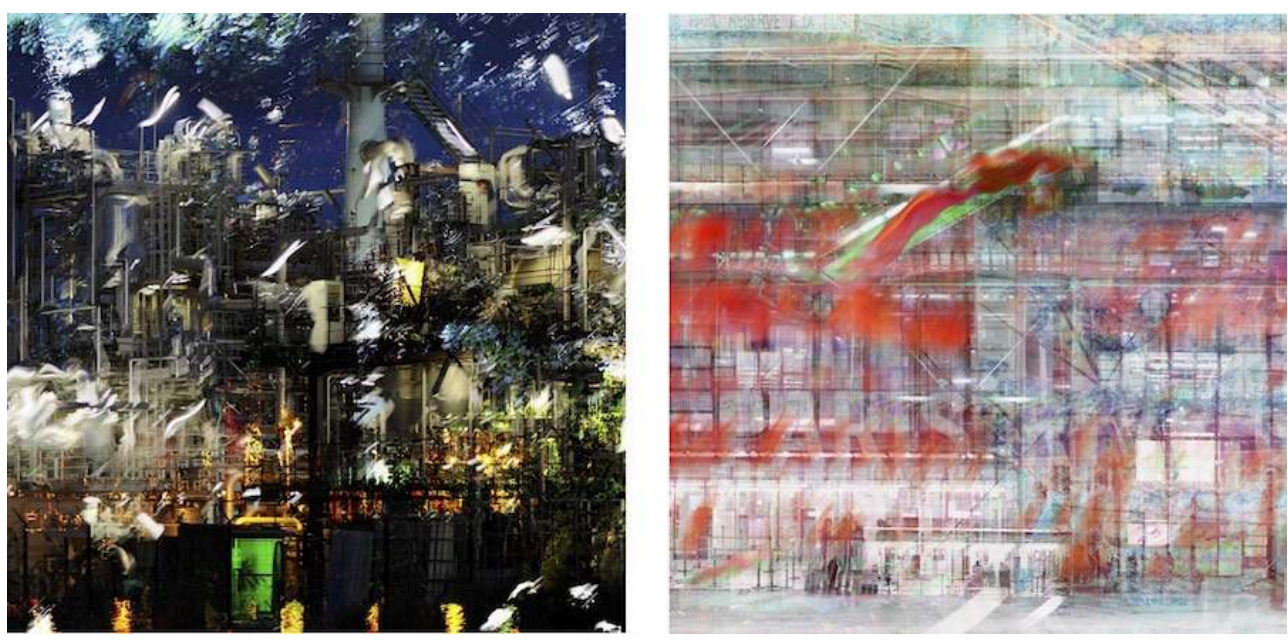

À gauche, lumières et reflets : superpositions, Le Havre (76) ; à droite, paysage urbain, extraction, La Défense à Paris.

Notre recherche patiente se teste également en zone urbaine.

La friche industrielle du Havre est une très belle zone de reconquête végétale, malgré les usines classées Seveso. Le travail photographique s'effectue de nuit, quand les installations lumineuses crachent leurs lumens et éclairent brutalement ces prairies sauvages servant de zone non aedificandi et qui protègent les activités industrielles les unes par rapport aux autres.

47 Autre lieu, autre recherche. Les immeubles de La Défense, tout en vitre et en façade, reflètent inlassablement des espaces publics. Notre travail photographique met en avant ces effets de reflets, montre la multiplicité des lumières, des vides et des creux de la ville. L'espace public comme salle d'exposition à ciel ouvert... si on le regarde autrement. Là encore modifier le regard que l'on porte, soi-même, et montrer à nos interlocuteurs une nouvelle façon de voir la ville.

Grâce à ce travail d'herbiers de paysage, de collections de croquis, de photomontages, notre sensibilité aux aspects extraordinaires du monde ordinaire qui nous entoure s'exacerbe, nous rend réceptif. Cette sensibilité accrue nous apporte une manière plus personnelle d'appréhender les questions de «nature » en ville, de l'aménagement des espaces ouverts urbains. Les propos sur la diversité que nous défendons en tant que paysagistes s'illustrent et s'organisent. Ces images collectées, retravaillées, fabriquées nous permettent de faire partager cette vision esthétique et nos projets de paysage s'enrichissent de cette sensibilité. Nos discours et propositions sont plus fluides, plus ouverts à l'appropriation, plus poétiques. La compréhension du projet se fait là, par l'approche sensible et partagée de l'image. 


\section{BIBLIOGRAPHIE}

Beer, O., Lucien Hervé, l'homme construit, Paris, Le Seuil, 2001.

Crawford, A., Mario Giacomelli, New-York, Phaidon Press Limited, 2001.

Leiter, S., Early Color, Göttingen, Steild, 2006.

Moon, S., Coïncidences, London, Thames \& Hudson Ltd, 2001.

\section{RÉSUMÉS}

Dans le cadre de notre pratique professionnelle de paysagistes, nous sommes amenés à produire des images. Nous cherchons l'inspiration en amont des projets. Comme le botaniste collectionne les herbes et les plantes qu'il trouve, nous collectionnons les paysages parcourus, traversés ou analysés.

Cette collecte donne lieu a des « herbiers de paysage " mêlant données prises sur le vif (photos, croquis, feuilles) et images recomposées. C'est ce travail sur les données brutes et données transformées structurant notre part d'invention dans le projet que nous souhaitons commenter ici.

Entre la prolifération des images, les nouveaux outils de l'informatique, la facilité des montages et photomontages, le brouillage des idées est là tout autour de nous. Nous avons besoin de cette recherche patiente en amont des projets pour renouveler notre inspiration et alimenter nos points de vue. C'est ce travail des images qui donne à voir et révèle les invisibles. C'est ce travail d'images qui incite à revenir sur le terrain. C'est également ce travail sur le flou des images fabriquées qui alimente le débat dans la fabrique partagée de nos projets de paysage.

In our professional practice as landscape architects, we produce images. and seek inspiration before starting projects. As the botanist collects the herbs and plants he finds, we collect the landscapes we have visited, travelled through and studied. These collections which combine materials taken directly from the field (photos, sketches, leaves) and recomposed images constitute as many "landscape herbaria". In this article we comment on this work we carry out on the raw and transformed data which gives form to our creative input in landscape projects. The proliferation of images, the availability of new computer tools, and the ease with which it is possible to edit images and produce photomontages results in a blurring of ideas. Before embarking on projects, we need to conduct patient research to find new inspiration and fresh insights. This work on images enables us to perceive and represent elements which are invisible and motivates us to go back into the field. It is also this work on the blurred nature of fabricated images that fuels the debate in the shared production of our landscape projects.

\section{INDEX}

Mots-clés : herbiers de paysage, représenter le vivant, catalogue d'images, conception, images floues

Keywords : landscape herbaria, representing life, image catalogue, design, blurred images 


\section{AUTEURS}

\section{FLORENCE ROBERT}

Paysagistes depuis plus de 20 ans nous travaillons sur divers projets avec différents partenaires, en équipes restreintes ou plus étoffées, sur des sites très urbains ou plus ruraux, dans différentes villes, différentes régions. Nous parcourons la France au hasard des commandes et des engagements pour réaliser jardins, aménagements, analyse de territoire, mais toujours autour de l'espace public ou partagé. Notre sensibilité nous amène à reconnaître les paysages et leurs diversités, à nous en inspirer aussi. Notre fascination pour le monde du végétal pousse notre curiosité dans une recherche de solutions esthétiques, économiques et pérennes. Notre travail se double d'une recherche sur la représentation d'une flore plus indépendante des jardiniers, moins horticole, peut-être moins appréciée, et pourtant expressive. Faire avec la nature, plutôt que contre, nous oblige à comprendre les dynamiques des écosystèmes, à changer notre regard pour apprécier une végétation plus autonome, plus « sauvage ».

contact[at]rb-cie[dot]net

www.rb-cie.net

\section{FRÉDÉRIC BCEUF}

Paysagistes depuis plus de 20 ans nous travaillons sur divers projets avec différents partenaires, en équipes restreintes ou plus étoffées, sur des sites très urbains ou plus ruraux, dans différentes villes, différentes régions. Nous parcourons la France au hasard des commandes et des engagements pour réaliser jardins, aménagements, analyse de territoire, mais toujours autour de l'espace public ou partagé. Notre sensibilité nous amène à reconnaître les paysages et leurs diversités, à nous en inspirer aussi. Notre fascination pour le monde du végétal pousse notre curiosité dans une recherche de solutions esthétiques, économiques et pérennes. Notre travail se double d'une recherche sur la représentation d'une flore plus indépendante des jardiniers, moins horticole, peut-être moins appréciée, et pourtant expressive. Faire avec la nature, plutôt que contre, nous oblige à comprendre les dynamiques des écosystèmes, à changer notre regard pour apprécier une végétation plus autonome, plus « sauvage ».

contact[at]rb-cie[dot]net

www.rb-cie.net 